\title{
Performance of the BACCUS Transition Radiation Detector
}

N. Picot-Clémente ${ }^{*+1}$, Y. Amare ${ }^{1}$, T. Anderson ${ }^{2}$, D. Angelaszek ${ }^{1,3}$, N. Anthony ${ }^{3}$, K. Cheryian $^{3}$, G.H. Choi ${ }^{4}$, M. Copley ${ }^{1}$, S. Coutu' ${ }^{2}$, L. Derome ${ }^{5}$, L. Eraud ${ }^{5}$, L. Hagenau ${ }^{3}$, J.H. Han ${ }^{1}$, H.G. Huh ${ }^{1}$, S. Im ${ }^{2}$, J.A. Jeon ${ }^{4}$, S. Jeong ${ }^{4}$, K.C. Kim ${ }^{1}$, M.H. Kim ${ }^{1}$, H.Y. Lee ${ }^{4}$, J. Lee ${ }^{4}$, M.H. Lee ${ }^{1}$, J. Liang ${ }^{3}$, J.T. Link ${ }^{6}$, L. Lu ${ }^{3}$, L. Lutz ${ }^{1}$, A. Menchaca-Rocha ${ }^{7}$, T. Mernik $^{1}$, J.W. Mitchell ${ }^{6}$, S.I. Mognet ${ }^{2}$, S. Morton ${ }^{3}$, M. Nester ${ }^{3}$, S. Nutter ${ }^{8}$, O. Ofoha ${ }^{1}$, I.H. Park ${ }^{4}$, R. Quinn ${ }^{3}$, E.S. Seo ${ }^{1,3}$, J.R. Smith ${ }^{1}$, P. Walpole ${ }^{1}$, R.P. Weinmann ${ }^{1}$, J.Wu ${ }^{1}$, Y.S. Yoon ${ }^{1}$

1 Inst. for Phys. Sci. and Tech., University of Maryland, College Park, MD 20742, USA

2 Dept. of Physics, Penn State University, University Park, PA 16802, USA

3 Dept. of Physics, University of Maryland, College Park, MD 20742, USA

4 Dept. of Physics, Sungkyunkwan University, Suwon 440-746, Republic of Korea

5 Laboratoire de Physique Subatomique et de Cosmologie, UJF - CNRS/IN2P3 - INP, 53 rue des Martyrs, 38026 Grenoble Cedex, France

6 Astroparticle Physics Laboratory, NASA Goddard Space Flight Center, Greenbelt, MD 20771, USA

7 Instituto de Fisica, Universidad Nacional Autonoma de Mexico, Circuito de la Investigación s/n, Ciudad Universitaria, CP 04510 México Distrito Federal, Mexico

8 Dept. of Physics and Geology, Northern Kentucky University, Highland Heights, KY 41099, USA

The Boron And Carbon Cosmic rays in the Upper Stratosphere (BACCUS) balloon-borne experiment flew for 30 days over Antarctica in December 2016. It is the successor of the CREAM balloon program in Antarctica which recorded a total cumulative exposure of 161 days. BACCUS is primarily aimed to measure cosmic-ray boron and carbon fluxes at the highest energies reachable with a balloon or satellite experiment, in order to provide essential information for a better understanding of cosmic-ray propagation in the Galaxy. The payload is made of multiple particle physics detectors which measure the charge up to $\mathrm{Z}=26$ and energy of incident particles from a few hundred GeV to a few PeV. The newly designed Transition Radiation Detector (TRD) measures signals that are a function of the charge and Lorentz factor. In April 2016, BACCUS was taken to CERN in its flight configuration to characterize its detectors' response to beams of electrons and pions. The performance of the TRD using beam test data are reported in this paper.

35th International Cosmic Ray Conference - ICRC2017-

10-20 July, 2017

Bexco, Busan, Korea

\footnotetext{
*Speaker.

${ }^{\dagger}$ Email: picot@umd.edu
} 


\section{Introduction}

The balloon-borne BACCUS mission is the successor of the CREAM program in Antarctica, which recorded 161 days of cumulative exposure after six long duration balloon flights from 2004 to 2010 [四]. BACCUS was flown in December 2016 for 30 days. It is primarily aimed to provide measurements of the boron and carbon fluxes at the highest energies possible.

Boron particles are mainly secondary cosmic rays created after interaction of primary carbon particles in the interstellar medium. Measurements of the secondary-to-primary ratio B/C give thus direct information on the amount of matter traversed by cosmic rays during their propagation in the Galaxy.

The BACCUS instrument is a suite of particle detectors that allow us to measure cosmic rays from a few hundred $\mathrm{GeV}$ to a $1 \mathrm{PeV}$, and can identify nuclei from protons of charge $\mathrm{Z}=1$ to iron of charge $Z=26$. The newly designed gas Transition Radiation Detector (TRD) is aimed to provide Lorentz factor measurements in the region of $\gamma \sim 10^{2}$ to $\gamma \sim 10^{5}$ for charged nuclei from $Z=3$ to $\mathrm{Z}=26$. The TRD brings the possibility to perform inter-calibration with energy measurements from the calorimeter, and is expected to further increase the accuracy and sensitivity of the instrument.

In April 2016, the whole BACCUS instrument was shipped to CERN in order to characterize the performance of its detectors in their flight configuration. The response to beams of electrons and pions at multiple energies was measured for the various detectors. In Section $\S \square$ and $\S$, the BACCUS instrument are presented and the CERN beam test configuration are described. The response of the newly designed TRD to beams of electrons and pions are reported in Section $\S$ 田.

\section{The BACCUS Instrument}

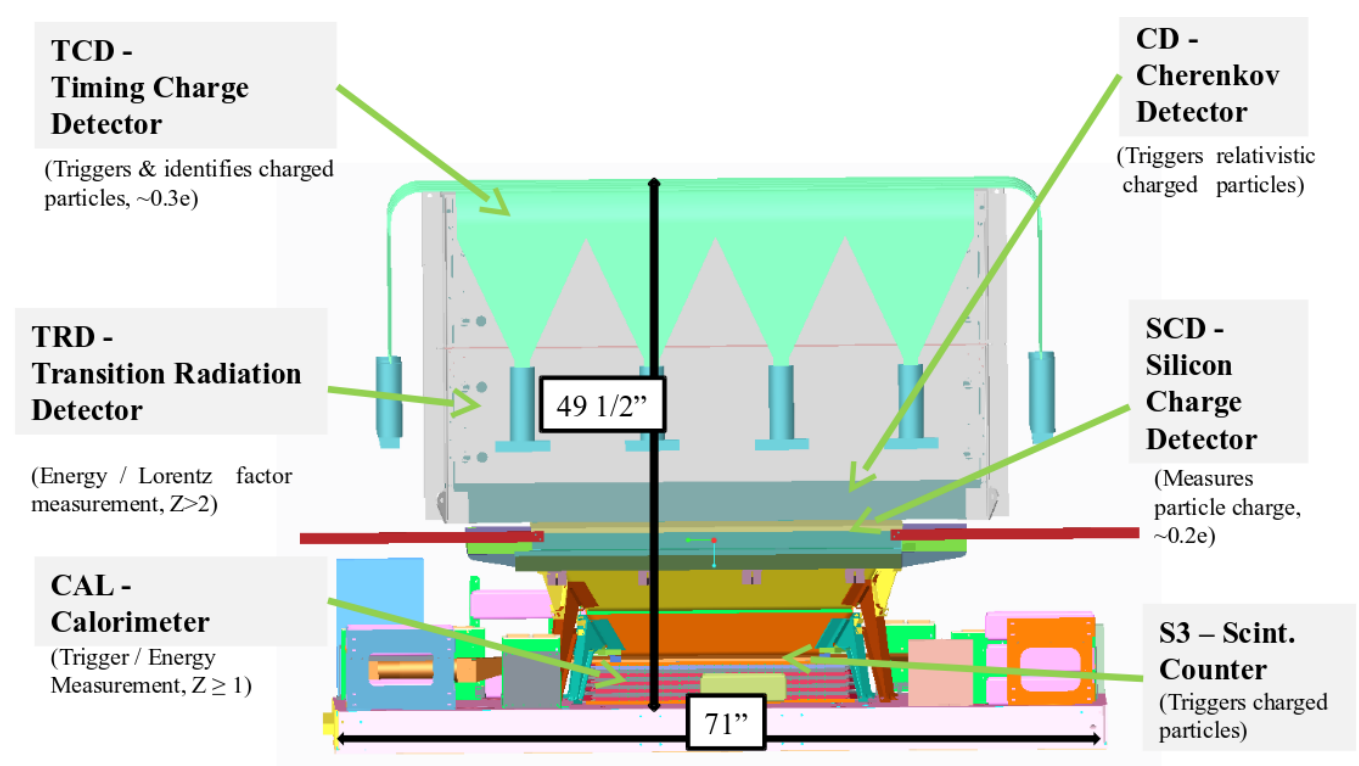

Figure 1: Schematic view of the BACCUS instrument.

The BACCUS instrument consists of redundant and complementary particle detectors that can

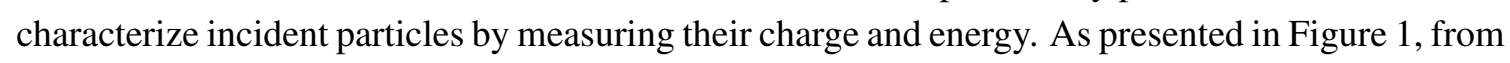


top to bottom, a downward-going particle will cross the Timing Charge Detector (TCD), the TRD, the Cherenkov Detector (CD), two layers of Silicon Charge Detector (SCD) and the Calorimeter (CAL). The TCD provides trigger information by detecting the light produced by charged particles in plastic scintillators. The $\mathrm{CD}$ is used to reject low-energy particles that don't produce Cherenkov light in the plastic of refractive index 1.5. The finely segmented SCD can differentiate cosmic-ray nuclei from $Z=1$ to $Z=26$. The 20-radiation length $C A L$ is made of 20 tungsten plates interleaved with scintillating fiber ribbons and fiber-glass sheets, and is designed to measure the energy of incident nuclei from a few hundred $\mathrm{GeV}$ to $1 \mathrm{PeV}$.

A schematic view of the TRD is presented in Figure $\square$. An initial TRD Monte Carlo study,

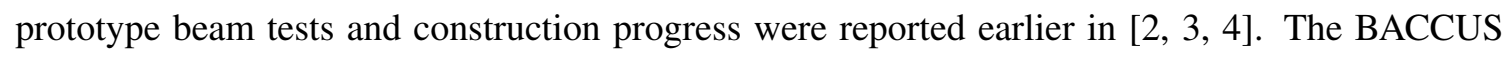
TRD is made of $5 \mathrm{~mm}$ radius proportional straw tubes which can greatly improve particle tracking, and thus charge identification with the SCD, as well as detector path-length corrections. The TRD is comprised of 8 modules each containing 200 straws alternating orthogonally in the $\mathrm{X}$ and $\mathrm{Y}$ direction, interleaved with $50 \mathrm{~mm}$ thick transition plastic radiators. Styrofoam polystyrene foam material was chosen for radiators, different from the polyethylene Ethafoam-220 foam material used in the CREAM-I TRD, that showed residual deformations after thermal-vacuum tests at the NASA Columbia Scientific Balloon Facility (CSBF).

In each layer, the 200 straws are arranged into dense double layers shifted by $0.4 \mathrm{~mm}$, and glued together. The tubes were fabricated with very thin $(35 \mu \mathrm{m})$ aluminized Mylar film by ultrasonic welding. Each straw tube is filled with a gas mixture of $\mathrm{Xe} / \mathrm{CO} 2(80 \% / 20 \%)$, which is known for its large relativistic rise in ionization energy loss []. The ionization produced on the particle path is proportional to the charge and energy of the cosmic-ray nuclei. Moreover, transition X-rays produced in the radiators by charged particles with enough Lorentz factor (at about $\gamma>10^{3}$ ) create additional ionization signals in the TRD straw tubes.

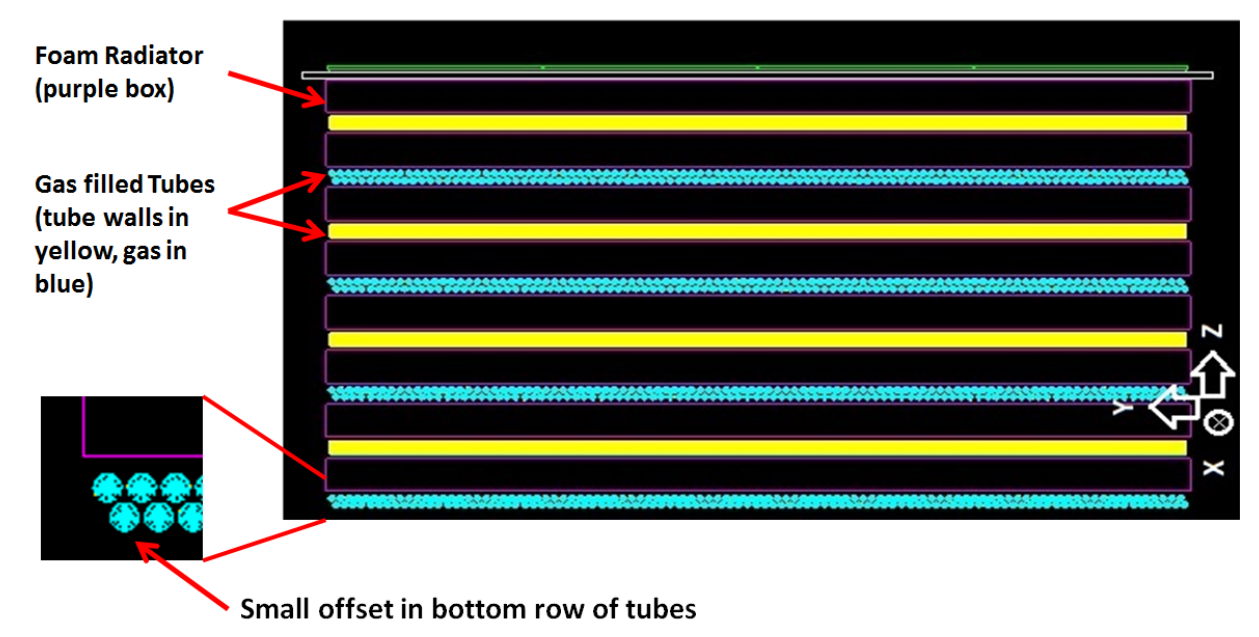

Figure 2: Schematic view of the TRD in the Y-Z plane.

\section{CERN Beam Test Configuration}

The whole BACCUS payload was shipped to CERN in April 2016. Figure [] shows the BAC- 
CUS instrument rotated by $90^{\circ}$ (laid on its side) on the moving table used for beam test in the $\mathrm{H} 2$ area of the CERN SPS secondary beam line.

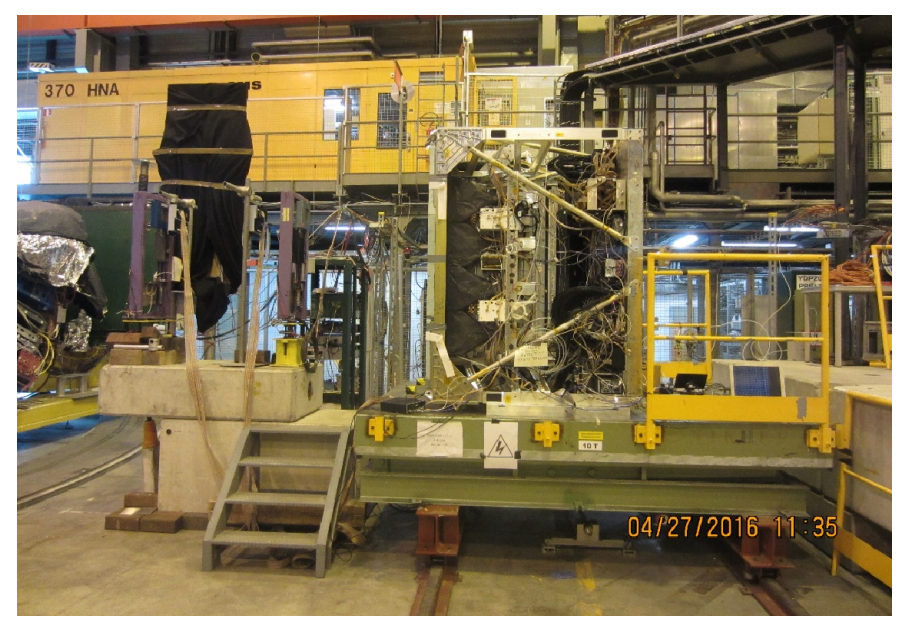

Figure 3: The BACCUS payload rotated by $90^{\circ}$ on a moving table in the $\mathrm{H} 2$ beam area at CERN.

Instead of $\sim 6$ days' worth of data taking, the beam ended up being available for $\sim 1.5$ days. This time constraint led to readjust measurement expectations to lower statistics and fewer positions. The response of the TRD to the energy of incident particles was measured using electron beams with energies from $20 \mathrm{GeV}$ to $200 \mathrm{GeV}$, and with pion beam energies from $100 \mathrm{GeV}$ to 300 $\mathrm{GeV}$ at three neighboring positions separated by $3 \mathrm{~mm}$. The uniformity of the TRD response was measured by translating the moving table horizontally and vertically while keeping the same 100 $\mathrm{GeV}$ electron beam.

During the test, the BACCUS instrument operated in its nominal flight configuration. The TRD tubes were filled with a Xe/CO2 gas mixture of $80 \% / 20 \%$ at a pressure of 1 atm. Layer 1 and 5 had gas leaks and high-voltage instabilities and were not operational during the beam test calibration.

\section{Response of the Transition Radiation Detector}

\subsection{Energy Scan}

The payload was exposed to beams of electrons, of purity between $\sim 90 \%$ and $\sim 95 \%$, in the energy range of $20 \mathrm{GeV}$ to $200 \mathrm{GeV}$ and pions, of purity close to $100 \%$, in the energy range of 100 $\mathrm{GeV}$ to $300 \mathrm{GeV}$. The instrument was moved to 3 adjacent positions separated by $3 \mathrm{~mm}$ to measure and account for possible geometric effect on the TRD signal. To check the quality of the beams, we used the known high linear response of the CAL to the electron beam energy [ [ $]$ ]. Figure 9 shows the energy deposit measured in the CAL as a function of the electron beam energy. The CAL presents a highly linear response as a function of the incident beam energy of $\sim 5.4 \mathrm{MeV} / \mathrm{GeV}$, so a sampling fraction of $\sim 0.54 \%$. This result confirmed the good quality of the beams used, in terms of energy and purity.

The TRD response (preliminary) as a function of Lorentz factor for incident electrons and pions is shown in Figure [5]. It is compared to previous measurements from 2010 with the same 


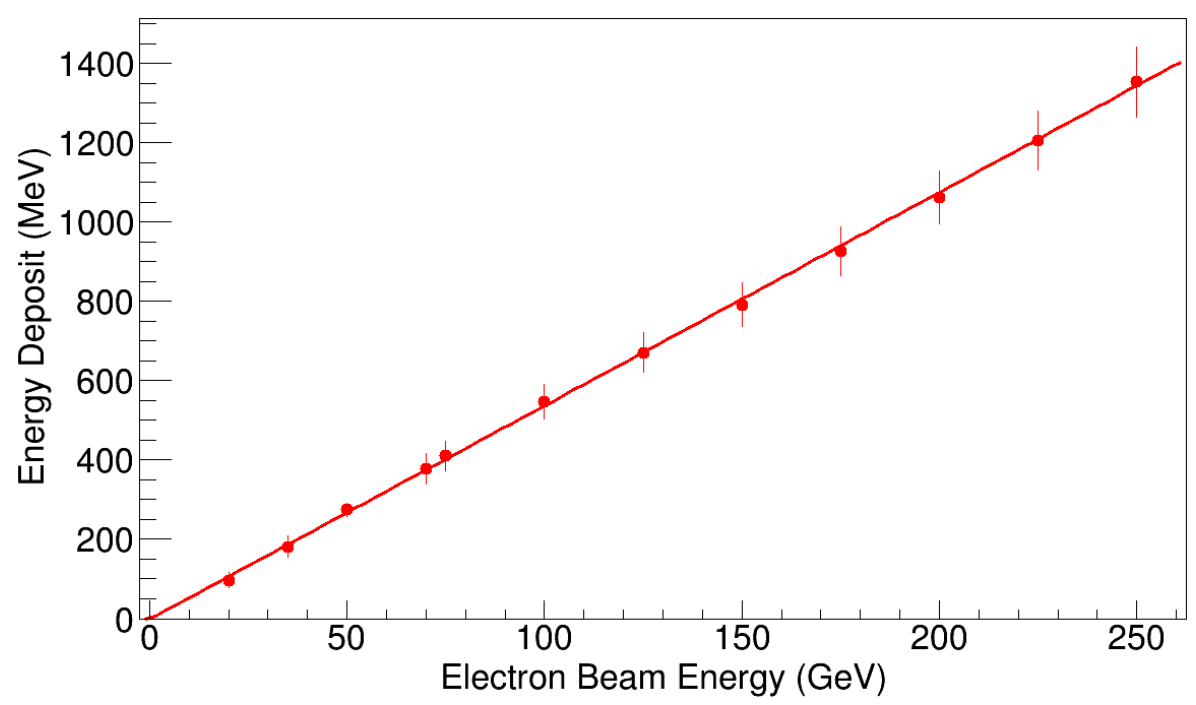

Figure 4: CAL energy deposit measured as a function of the electron beam energy. Data from the 2015 [ [6] and 2016 beam test are used here.

TRD [प], and to results from the CREAM-I TRD beam test and its associated predictions (see [8] for more details). The solid line corresponds to predictions obtained from GEANT4 Monte Carlo simulations [ $[\mathrm{Q}]$ using the "TranspR" transition radiation model, which generates transition radiation inside the foam radiator [ए0] 2016 beam test data are normalized in order to match predictions at $\gamma \sim 10^{3}$. As expected, measurements show that the emission of transition radiation at high $\gamma$ induces significantly higher signal in the TRD. Results from beam test data show a clear separation between signals from the pions (low $\gamma$ ) and the electrons (high $\gamma$ ).

The CREAM-I TRD was made of 8 layers composed of 512 straw tubes of $2 \mathrm{~cm}$ diameter embedded in polyethylene Ethafoam-220 foam radiator blocks. Tubes were filled with a $95 \%$ xenon 5\% methane gas mixture. Beam test measurements with the CREAM-I TRD show larger transition radiation signals than with the newly designed TRD, which is mainly due to the different foams and geometry used [ $[\mathbf{U}]$. Some discrepancies are observed between the 2010 and 2016 beam test data and are mainly due to different foams used (Ethafoam in 2010 and Styrofoam in 2016). Preliminary results are prior to any calibration corrections, needed to account for different highvoltage regimes and positions. Variations at high $\gamma$ are also due to electron beam contamination with hadrons and muons, which will be removed later using calorimeter measurements.

\subsection{Position Scan}

Due to beam time constraints, the energy scans presented above were always performed at the same 3 TRD neighboring positions. Figure 6 presents the event display of a $100 \mathrm{GeV}$ electron beam of $\sim 0.6 \mathrm{~cm}$ RMS width profile, crossing the TRD straw tubes oriented in the $\mathrm{X}$ direction. The figure shows how precisely the trajectory path of the particle beam can be determined with the TRD. Accurate track reconstruction can significantly reduce SCD charge measurement uncertainty by extrapolating the track to the detector layers. Similarly, as stated previously, a better knowledge 


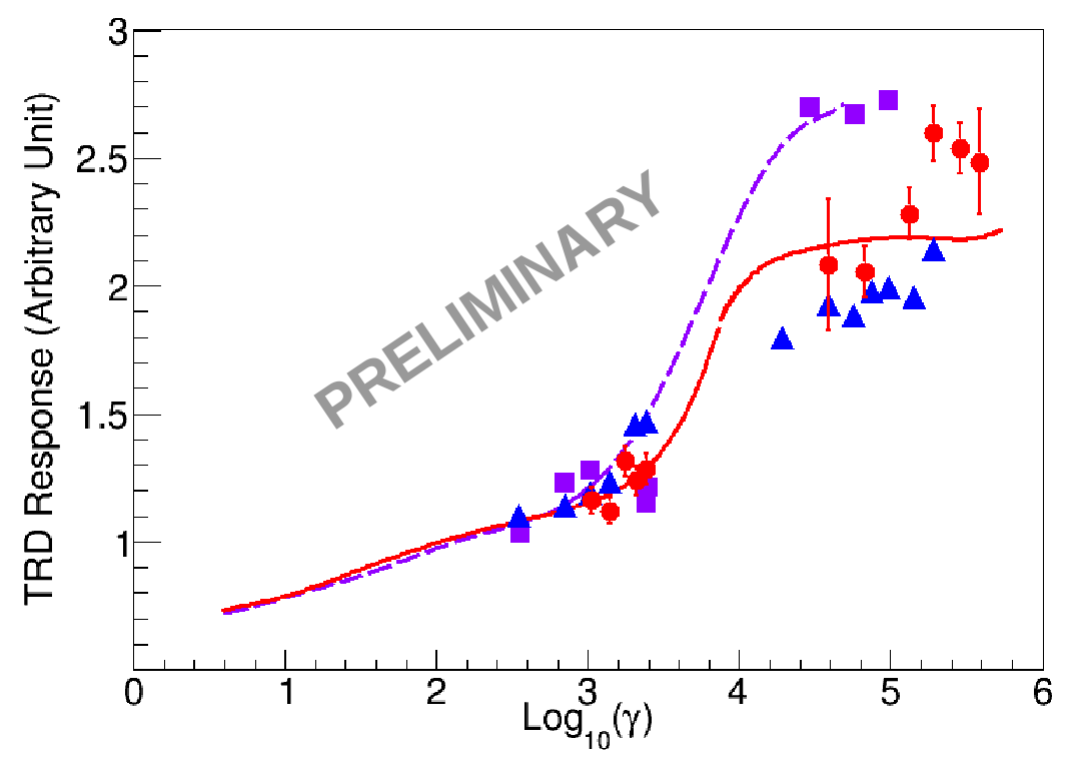

Figure 5: Preliminary BACCUS TRD response as a function of the Lorentz factor from 2016 beam test (red circle), compared to previous measurements from 2010 with same TRD but different foam (blue triangle) [D], and to predictions from GEANT4 Monte Carlo simulations (solid line) [Q] using the "TranspR" transition radiation model, which generates transition radiation inside the radiator [ए]]. Results from the CREAM-I TRD beam test (purple square) and its associated prediction curve (dotted line) are also shown [8]].

of the particle's path in the different layers of the instrument helps to compute more accurate energy loss corrections, which are used to calculate incident nuclei energies at the top of the instrument.

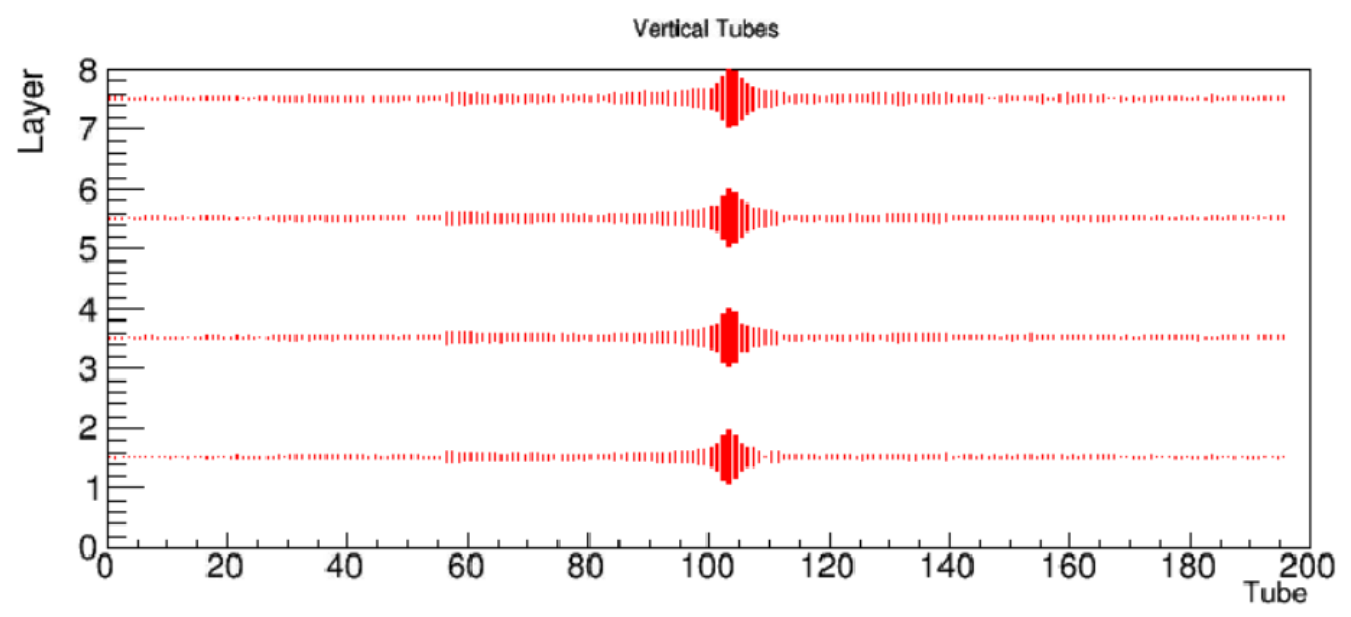

Figure 6: TRD event display of a $100 \mathrm{GeV}$ electron beam in the Y-Z plane of the detector.

To measure the uniformity of the TRD response, the moving table on which the BACCUS payload was placed, was moved every $1 \mathrm{~cm}$ horizontally and vertically. At each position, a beam of $100 \mathrm{GeV}$ electrons was used for measurements. The response variations are then used to calibrate each of the 1600 straw tubes. 


\section{Conclusions}

BACCUS flew in December 2016 for 30 days. It is primarily aimed to directly measure cosmic-ray boron and carbon nuclei at the highest energies to bring a better understanding of the cosmic-ray propagation history in the interstellar medium. The transition radiation detector is designed to measure the Lorentz factor of incident particles, and thus bring inter-calibration with energy measurements from the calorimeter. In April 2016, the whole BACCUS payload was calibrated at CERN in its flight configuration. The instrument was exposed to beams of electrons and pions, and the transition radiation expected at $\gamma \gtrsim 10^{3}$ was observed in the beam test. The detector presents a good tracking resolution, which can help reduce charge measurement uncertainty and compute more precise energy loss corrections in the instrument. In order to better characterize the detector's performance, each of the straw tube response is currently being calibrated with beam test data. In parallel, Monte Carlo simulations are being improved.

\section{Acknowledgments}

The authors thank the NASA Wallops Flight Facility, Columbia Scientific Balloon Facility, National Science Foundation Office of Polar Programs, and Raytheon Polar Services Company for the successful balloon launch, flight operations, and payload recovery for each balloon flight. They also thank CERN for provision of excellent accelerator beams. This work is supported in the U.S. by NASA grants NNX11AC52G, NNX08AC15G, NNX08AC16G and their predecessor grants, as well as directed RTOP funds to the NASA Goddard Space Flight Center. It is supported in Korea by the Creative Research Initiatives of MEST/NRF and by National Research Foundation Grants NRF-2014R1A2A2A01002734, NRF-2014R1A1A2006456. It is supported in France by IN2P3/CNRS and CNES and in Mexico by DGAPA-UNAM and CONACYT.

\section{References}

[1] E.S. Seo et al., Cosmic-ray energetics and mass (CREAM) balloon project, Adv. Sp. Res. 33, 10, 1777-1785 (2004).

[2] A. Malinin et al., Next Generation TRD for CREAM Using Gas Straw Tubes and Foam Radiators, Proc. 30th Int. Cosmic Ray Conf., Merida, Mexico, ID 346 (2007).

[3] A. Malinin et al., A New Transition Radiation Detector for the CREAM experiment, Proc. 31th Int. Cosmic Ray Conf., Łódź, Poland, ID 1033 (2009).

[4] A. Malinin et al., A New Transition Radiation Detector for the CREAM experiment, Proc. 32th Int. Cosmic Ray Conf., Beijing, China, ID 1223 (2011).

[5] H.S. Ahn et al., The Cosmic Ray Energetics And Mass (CREAM) instrument, Nucl. Inst. Meth. Phys. Res. A 579, 1034-1053 (2007).

[6] N. Picot-Clemente et al., Performance of the ISS-CREAM Calorimeter, Proc. 35th Int. Cosmic Ray Conf., Busan, Korea (2017).

[7] A. Malinin et al., A Transition Radiation Detector for the BACCUS experiment, Proc. 33rd Int. Cosmic Ray Conf., Rio, Brazil, ID 612 (2013). 
[8] S.P. Swordy, P. Boyle, S. Wakely, Comparison of a Transition Radiation Detector Response with Numerical Simulations, Proc. 28th Int. Cosmic Ray Conf., Tsukuba, Japan, 2233-2236 (2003).

[9] S. Agostinelli et al., Geant4 - A Simulation Toolkit, Nuc. Inst. and Meth. A 506, 250, 2003. J. Allison et al., Geant4 Developments and Applications, IEEE Trans. on Nuc. Science 53 (1), 270, 2006.

[10] Physics Reference Manual, http://geant4.web.cern.ch/geant4/UserDocumentation/UsersGuides/PhysicsReferenceManual/fo/PhysicsReferenceManual.pdf, 2016. 